\title{
Simple synthesis of large-area multilayer graphene films on dielectric substrate via chemical vapor deposition route (synthesis of MLG films on dielectric substrates via CVD route)
}

\begin{abstract}
A systematic study of three distinct process variables to optimize the maximum formation of multilayer graphene (MLG) thin films grown over an alumina substrate supported Co-Ni catalyst was performed. The three considered parameters were the reaction temperature, catalyst composition, and ethanol flow rate. The optimization process was employed to ensure a high performance in the utilized experimental ranges and to evaluate the interactive effects of the three parameters on the MLG yield of the ethanol-based chemical vapor deposition (CVD) method for potential gas-sensing applications. The synthesis and physical properties of the MLG characterized over the $0.3 \mathrm{Co}-0.7 \mathrm{Ni} / \mathrm{Al} 2 \mathrm{O} 3$ catalyst under optimum conditions were characterized using X-ray diffraction (XRD) (Rigaku; MiniFlex diffractometer with a $\mathrm{Cu}$ KŬ radiation source, $\approx 0.15418 \mathrm{~nm}$ ), field emission scanning electron microscopy (FESEM) (JSM-7800F) and Raman spectroscopy (RS) (Jobin Yvon Horiba HR800UV) analysis.
\end{abstract}

Keyword: Chemical vapor deposition; Dielectric substrates; Multilayer graphene; Response surface methodology 